Mens

revue d'histoire intellectuelle de l'Amérique française

MENS

\title{
La Revue moderne et le nationalisme, 1919-1920
}

\section{Jean-Christian Pleau}

Volume 6, numéro 2, printemps 2006

URI : https://id.erudit.org/iderudit/1024303ar

DOI : https://doi.org/10.7202/1024303ar

Aller au sommaire du numéro

Éditeur(s)

Centre de recherche en civilisation canadienne-française

ISSN

1492-8647 (imprimé)

1927-9299 (numérique)

Découvrir la revue

Citer cet article

Pleau, J.-C. (2006). La Revue moderne et le nationalisme, 1919-1920. Mens, 6(2), 205-237. https://doi.org/10.7202/1024303ar
Résumé de l'article

La Revue moderne fondée en 1919 par la journaliste Madeleine, a joué un rôle important dans la vie culturelle québécoise des années vingt. La dimension politique de cette revue a cependant été moins étudiée. Paraissant dans le contexte de l'après-guerre, et peu de temps après des crises qui avaient favorisé l'essor du nationalisme canadien-français, la revue de Madeleine prend nettement position en faveur de l'unité nationale et se signalera par des attaques contre le nationalisme et ses chefs de file. On analysera ici le discours politique véhiculé par les principaux collaborateurs de la revue en 1919-1920, et l'on examinera notamment le débat entre Arthur Beauchesne et Wilfrid Gascon sur la question de l’indépendance du Québec. 


\title{
LA REVUE MODERNE \\ ET LE NATIONALISME, 1919-1920
}

\author{
Jean-Christian Pleau \\ Département d'études littéraires \\ Université du Québec à Montréal
}

\section{Résumé}

La Revue moderne, fondée en 1919 par la journaliste Madeleine, a joué un rôle important dans la vie culturelle québécoise des années vingt. La dimension politique de cette revue a cependant été moins étudiée. Paraissant dans le contexte de l'après-guerre, et peu de temps après des crises qui avaient favorisé l'essor du nationalisme canadien-français, la revue de Madeleine prend nettement position en faveur de l'unité nationale et se signalera par des attaques contre le nationalisme et ses chefs de file. On analysera ici le discours politique véhiculé par les principaux collaborateurs de la revue en 1919-1920, et l'on examinera notamment le débat entre Arthur Beauchesne et Wilfrid Gascon sur la question de l'indépendance du Québec.

\section{Abstract}

Founded in 1919 by the journalist Madeleine, La Revue moderne played an important role in Quebec's cultural life in the 1920s. However, the political dimension of this magazine has not received the same level of attention from scholars. Appearing in the postwar context, shortly after a number of crises which encouraged the rise of French Canadian nationalism, Madeleine's magazine took a clear stand in favour of national unity and was noted for its attacks on nationalism and its leaders. The political discourse developed by the review's main uriters in 1919-1920 will be analysed here; particular attention will be given to the debate between Arthur Beauchesne and Wilfrid Gascon on the topic of Quebec'sindependence. 
La Revue moderne, mensuel fondé par la journaliste Madeleine ${ }^{1}$, fut l'un des titres importants de la presse populaire québécoise de 1919 à 1960. La relative longévité de ce périodique - et l'apparente stabilité à laquelle celle-ci pourrait faire croire - ne doit pas dissimuler le fait que la revue subit des transformations au cours de son histoire: devenue une revue populaire à fort tirage dans les années quarante, elle eut au départ de hautes ambitions littéraires et intellectuelles. À en croire son sous-titre ("Littérature. Politique. Arts»), la revue entendait concurrencer les périodiques littéraires ou politiques les plus sérieux, tel l'austère Canada français de l'Université Laval. Mais par la présentation soignée des livraisons, par le luxe affiché dans la publicité ou dans les pages féminines - qui sont en soi un témoignage fascinant sur une certaine prospérité d'après-guerre -, par la place accordée à la littérature romanesque ${ }^{2}$, il est clair que la revue cherchait surtout à séduire un lectorat bourgeois. Jusque vers 1924 , on peut dire que La Revue moderne a rempli avec succès ce double mandat intellectuel et mondain - à tout le moins, autant qu'il pouvait l'être dans le contexte de l'époque. Pourtant, les premières années de la revue n'ont jusqu'à présent que peu retenu l'attention des chercheurs. Parmi les spécialistes de la littérature, François Ricard, par exemple, a consacré une étude à la revue, mais pour la période des années trente ${ }^{3}$. Plus récemment, un groupe de recherche de l'Université Laval (Denis Saint-Jacques, Marie-José Des Rivières et Kenneth Landry) s'est penché de manière intéressante sur la première décennie de la revue, dans une perspective d'histoire culturelle - sans toutefois analyser spécifiquement le discours politique de celle$\mathrm{ci}^{4}$. Du côté des historiens des idées, la revue ne semble guère avoir suscité plus de curiosité. Il n'en était pas question par exemple dans les Idéologies an Canada frangais. ${ }^{5}$ Yvan Lamonde, pour sa part, la mentionne dans son Histoire sociale des idées au 
Québec, notamment à propos de la querelle entre régionalistes et exotiques, mais sans s'y attarder. Ce n'est en fait que dans Passage de la modernité de la sociologue Andrée Fortin que l'on trouvera une brève évocation du discours politique de La Revue moderne ${ }^{7}$. Toutefois, le propos par définition général de ce dernier ouvrage ne lui permettait pas d'effectuer une analyse détaillée du discours véhiculé par la revue de Madeleine; de fait, Andrée Fortin ne cite que le premier éditorial de la re$v^{2} e^{8}$. On peut estimer qu'une telle vision en survol, mais surtout l'absence de références à un corpus plus étoffé, l'entraînent à gauchir quelque peu les paroles de Madeleine dans le sens du nationalisme, alors que l'intention de celle-ci, comme on tâchera de le montrer ici, était plutôt de critiquer ce dernier et d'en appeler à un idéal de concorde et d'union pancanadienne? ${ }^{9}$. En somme, pour saisir le sens et la portée du discours politique véhiculé par la revue, une analyse plus ciblée et plus approfondie s'impose. On n'entreprendra donc pas ici de brosser un tableau complet des activités de la revue (ne fût-ce que pendant sa première décennie : ce serait une tâche par trop considérable, qui d'ailleurs intéresserait aussi bien l'histoire culturelle que celle des mœurs et des mentalités). L'objet de la présente étude sera plus circonscrit : il s'agira de montrer comment la revue, sous l'inspiration de Madeleine, se veut, dès sa fondation, un lieu où cherche à s'élaborer un discours politique sur la situation canadienne faisant en quelque sorte contrepoids aux divers discours nationalistes. Plus précisément encore, nous nous intéresserons ici aux tout premiers numéros de la revue ${ }^{10}$, ceux qui définissent en quelque sorte le ton que Madeleine voulait imprimer à $L a$ Revue moderne, et que celle-ci maintiendrait jusque vers $1924^{11}$. 


\section{Madeleine, personnalité méconnue}

Bien que l'on s'accorde généralement à souligner l'importance de la figure de Madeleine en tant que pionnière du journalisme féminin au Québec ${ }^{12}$, il faut convenir que celle-ci demeure en fait très mal connue. Aucune étude d'envergure ne lui a encore été consacrée, et des pans entiers de sa carrière demeurent dans l'ombre ${ }^{13}$. Ce désintérêt s'explique de plusieurs manières. D'une part, comme il arrive généralement quand on a affaire à des écrivains qui furent essentiellement des journalistes, il faut en premier lieu tenir compte de la difficulté matérielle qu'il y a à reconstituer une œuvre éparpillée dans un grand nombre de revues et de quotidiens : une toute petite partie seulement des articles de Madeleine a été rassemblée en recueil ${ }^{14}$. À quoi s'ajoute évidemment la difficulté pour une voix féminine de se faire entendre dans le contexte du début du vingtième siècle, a fortiori lorsqu'elle s'éloignait du champ traditionnel des intérêts féminins : préjugé qui pourrait suffire à expliquer non seulement l'hostilité parfois rencontrée par Madeleine de son vivant (elle fut l'objet d'une attaque féroce de Valdombre en $1940^{15}$ ), mais aussi l'oubli assez rapide dont elle fut l'objet après sa mort. Madeleine en effet prit sa retraite comme directrice de La Revue moderne en 1930, et interrompit alors presque complètement son activité journalistique. Il serait certes exagéré de dire qu'elle fut immédiatement oubliée : la publication en 1938 de ses Portraits de femmes suggère plutôt que Madeleine avait conservé son réseau de relations mondaines et qu'elle jouissait encore de l'appui de très hautes personnalités (tel le sénateur Dandurand, qui en rédigea la préface) : vers 1940, elle était encore reconnue comme une figure publique notable, - ce que l'attaque même de Valdombre confirmerait. Mais il est indéniable qu'elle était devenue beaucoup plus effacée, et que sa mort n'attira pas l'attention ${ }^{16}$. On aurait pu imaginer qu'un tel parti 
pris de désintérêt aurait été corrigé par des travaux récents d'inspiration féministe: mais il se trouve que la position de Madeleine sur des enjeux tels que le droit de vote des femmes était souvent d'un conservatisme prudent. En ce qui concerne l'histoire de la question féminine au Québec, Madeleine se trouve donc inévitablement éclipsée par des contemporaines plus audacieuses, qui ont jusqu'ici accaparé l'attention. Par ailleurs, il faut bien admettre que le mérite proprement littéraire des écrits de Madeleine n'était pas de nature à en assurer la survie, une fois oublié le contexte historique qui leur avait donné naissance ${ }^{17}$. D'où le paradoxe : une personnalité ayant occupé des tribunes de premier plan pendant une trentaine d'années - et l'une des premières voix féminines dans le journalisme au Québec — qui n'a cependant laissé presque aucune trace dans la mémoire collective, et qui commence à peine à être étudiée méthodiquement par les chercheurs universitaires.

Pour le petit nombre de ceux qui ont aujourd'hui une certaine familiarité avec ses écrits, Madeleine est surtout connue pour s'être illustrée dans des genres traditionnellement féminins. Le "courrier du cœur", d'abord à La Patrie puis à La Revue moderne, fut en effet l'une de ses grandes spécialités. De même, ses contes et autres pages littéraires publiés en volume (et par là mieux connus) privilégient un registre sentimental et moralisateur qui ne paraît pas s'écarter des conventions du domaine féminin telles qu'on pouvait les concevoir au début du siècle. Ce qui cependant n'a guère été examiné jusqu'ici, c'est l'importance du rôle de Madeleine en tant que directrice de La Revue moderne. Dans cette position, déjà inhabituelle à l'époque pour une femme, Madeleine, par ses éditoriaux, s'aventurait très loin de l'espace féminin et n'hésitait pas, par exemple, à prendre parti sur des questions politiques. D'une manière tout aussi importante, Madeleine jouait le rôle 
d'animatrice d'une sorte de cénacle d'écrivains qui partageaient sa sensibilité, notamment sur les questions de politique et d'identité culturelle ${ }^{18}$. Ce petit groupe littéraire gravitant autour de Madeleine constituait une sorte d'« école" de La Revue moderne, qui s'affirma notamment par des polémiques vigoureuses avec les écrivains nationalistes. N'entendons point par là une école consciente de sa propre existence et s'affichant comme telle (puisque nul ne s'en réclama jamais). Si la notion d'« école » a ici un sens, c'est parce que ces personnalités - généralement issues de la bourgeoisie mondaine - défendaient dans la revue un petit nombre d'idées communes, mais surtout parce qu'elles appartenaient, pour autant qu'on en puisse juger aujourd'hui, à un même réseau de sociabilité centré autour du salon de Madeleine ${ }^{19}$. Parmi ces écrivains, on remarquera quelques noms familiers aux historiens de la littérature : par exemple Louvigny de Montigny ou Louis Dantin. Le premier, ami de longue date de Madeleine, fut l'un des collaborateurs réguliers de la revue dès sa fondation. Dantin, pour sa part, collabora à la revue surtout dans les années trente, donc après le départ de Madeleine. Exilé aux États-Unis, il ne pouvait d'ailleurs entretenir avec Madeleine et ses amis des liens bien étroits. Si on peut le ranger dans «l'école» de La Revue moderne des années vingt, c'est parce qu'il y fit paraittre un article sur Chez nos ancêtres de l'abbé Groulx, - article empreint de réserves, qui constitua en quelque sorte la première escarmouche directe de la revue avec l'abbé (il y en aura beaucoup d'autres). Parmi les autres collaborateurs de la revue, certains sont plus obscurs mais mériteraient d'être mieux connus, - tel Arthur Beauchesne, dont il sera ici longuement question; d'autres enfin n'ont d'importance que ponctuelle, du moins en ce qui concerne l'histoire intellectuelle du Québec, - tel René du Roure, qui fut l'un des fers de lance de la campagne de La Revue moderne contre 
l'abbé Groulx. Professeur de littérature française à McGill et diplomate attaché au Consulat de France à Montréal, ce dernier en effet n'est plus guère connu aujourd'hui que comme celui qui, en attaquant Groulx dans La Revue moderne en 1922, a lancé la querelle de L'Appel de la race. On le devine très proche de Madeleine et de son réseau mondain (son nom est mentionné avec éloges dans la revue en toute sorte d'occasions), mais aucun de ses autres articles - d'ailleurs peu nombreux — n'eut le même retentissement que celui sur Groulx. Mais il faudrait d'ailleurs noter qu'au sein du cénacle de Madeleine, l'entente n'était pas toujours parfaite : ce fut le cas, par exemple, lorsque Olivar Asselin, collaborateur régulier des débuts (au point d'apparaitre comme l'un des animateurs de la revue), prit position contre ses collègues dans la querelle de L'Appel de la race et s'afficha vigoureusement comme défenseur de l'abbé Groulx ${ }^{20}$. Personnalité complexe, Asselin restait proche des nationalistes, mais s'était néanmoins un peu séparé d'eux par sa participation à la guerre et par sa francophilie. De fait, il conviendrait de souligner l'importance de la guerre quant aux positions politiques de la plupart des intellectuels envisagés dans la présente étude : Madeleine ou Montigny, par exemple, avaient été aussi nationalistes qu'Asselin au début du siècle. Mais l'attitude de Bourassa pendant la guerre heurta si violemment leur francophilie qu'elle les détourna durablement du nationalisme. Quoi qu'il en soit, l'important est que Madeleine nous apparait ici au centre d'un réseau politique et littéraire qui s'exprime à travers la revue - sans toutefois se confondre avec elle. Très curieusement, cet aspect capital de la carrière de Madeleine, qui aurait dû lui valoir le plus d'attention, est pourtant celui qui a été jusqu'à présent le plus frappé d'oubli. 


\section{Le programme politique de La Revue moderne}

Pour mesurer la hauteur des ambitions de Madeleine pour La Revue moderne et prendre en même temps connaissance des points essentiels de son "programme " politique, il suffit de lire son éditorial dans la première livraison de la revue, en novembre 1919. Le titre, qui devait devenir la devise de la revue, est déjà programmatique : «S'unir pour grandir ». Les premières phrases de l'éditorial ne laissent aucun doute sur la portée politique que Madeleine comptait donner à cette devise : « Notre pays aspire à de hautes destinées. C'est son droit impérieux. Mais pour atteindre aux sommets convoités, une union s'impose entre les races, les classes, les groupes ${ }^{21}$.» Il faut se reporter au contexte historique pour mesurer la charge polémique d'une déclaration qui, en d'autres circonstances, n'aurait sans doute été reçue que comme un simple poncif ou un vœu pieux. Mais l'allusion à l'union entre les « races $^{22}$ », en 1919, ne pouvait être perçue comme innocente. Il faut se souvenir que toute la période de la Première Guerre mondiale avait été l'occasion d'une polarisation extrême entre le Canada français et le Canada anglais. D'une part, il y avait eu la querelle scolaire ontarienne liée au fameux règlement XVII (1912-1927), qui rendait presque impossible l'enseignement français en Ontario. Puis il y avait eu les élections fédérales de décembre 1917, qui reportèrent au pouvoir le ministère unioniste (essentiellement conservateur) de Borden contre le vote unanime du Canada français, qui se voyait de ce fait privé de toute représentation au sein du gouvernement (le seul ministre francophone, Blondin, devait sa survie à un siège au Sénat). Ces derniers événements avaient eux-mêmes donné lieu à la motion de Joseph-Napoléon Francœur, député libéral à l'Assemblée législative du Québec, qui avait propulsé la notion d'indépendance du Québec dans le débat public en décembre 1917 et janvier 1918. Bien sûr, il faudrait 
encore mentionner la crise de la conscription et tout le mouvement nationaliste regroupé autour d'Henri Bourassa et du Devoir ${ }^{23}$. En un mot, l'appel lancé par Madeleine en décembre 1919 se faisait précisément sur la toile de fond de ce passé récent de querelles de "races » exacerbées, qu'il convenait à ses yeux d'oublier: "Déjà, chez nous, s'affirme un besoin d'oublier les discussions anciennes, d'abolir les vieilles rancunes, d'effacer les mauvais souvenirs, pour commencer une vie nationale, inspirée uniquement des intérêts canadiens, des progrès canadiens, des demains canadiens ${ }^{24}$." Pour les lecteurs, il ne pouvait y avoir le moindre doute : par son premier éditorial, La Revue moderne signalait déjà qu'elle prendrait position contre le nationalisme.

Cela dit, il n'était pas question de faire de La Revue moderne une publication ouvertement partisane, et encore moins l'organe d'un parti politique, - au contraire de tant d'autres publications de cette époque (cette situation concernait d'ailleurs surtout les quotidiens, beaucoup plus que les magazines mensuels comme La Revue moderne, lesquels obéissaient plutôt à une logique commerciale). La fortune du $\mathrm{D}^{\mathrm{r}}$ Wilfrid Huguenin, le mari de Madeleine, pouvait d'ailleurs assurer une relative indépendance matérielle à la revue. Cette indépendance est soulignée dans une note de la direction, dans la livraison du 15 février 1920 : «La Revue Moderne [sic] n'étant à la dévotion d'aucune politique, ni subventionnée par l'argent de qui que ce soit, peut permettre à ses écrivains d'exprimer sincèrement leurs idées, quitte à s'attirer des répliques qui mettront de la vie et de la pensée dans nos pages: "De la discussion jaillit la lumière", ne l'oublions pas $^{25}$. »

L'intention affichée par Madeleine dans son premier éditorial était plutôt de faire de la revue une tribune ouverte à toutes les tendances intellectuelles - et donc à toutes les orientations politiques - mais toujours avec cette exclusive 
qui, en termes discrètement voilés, parait bien viser les tenants du nationalisme :

L'intérêt national ne devrait-il pas déterminer un effort en commun, où seraient oubliées les haines de race, où seraient abolies les dissensions anciennes, et où l'on apprendrait vraiment à se connaître et à se comprendre. La fondation de ce centre de pensée canadienne s'impose, et la Revue Moderne [sic] veut aider à sa création.

Elle ouvre donc largement ses colonnes à tous ceux qui sont appelés, par leur talent et leurs études, à jouer le rôle dans la vie canadienne, de diffuser des idées, de promouvoir des progrès, et d'orienter des mouvements. Elle veut ainsi former une opinion saine et juste qui professera le mépris du préjugé et répudiera toute propagande de haine et de fanatisme $e^{26}$.

Cela explique l'éclectisme affiché dans la liste des collaborateurs figurant en tête du premier numéro, qui est pratiquement un annuaire littéraire et intellectuel du Canada français de l'époque. On y retrouve en effet aussi bien des hommes que des femmes, des religieux que des laïs, des conservateurs que des libéraux, et même quelques anglophones célèbres pour leurs efforts en faveur de la bonne entente, tels William Henry Moore et John Boyd ${ }^{27}$. Cette liste doit évidemment être prise avec un grain de sel : la plupart de ces « collaborateurs » ne donnèrent aucun texte à la revue, et il faut supposer que leur appui s'est borné à permettre la publication de leur nom à des fins de publicité. Un petit nombre d'entre eux insistèrent d'ailleurs pour le faire retirer dans les numéros suivants : d'où l'on peut conclure que cette liste n'avait pas été compilée après une consultation bien approfondie, et que certains noms avaient été inclus un peu à la légère. N'empêche : parmi les collaborateurs véritables de Madeleine, on trouve des sympathisants du Parti libéral (par exemple Olivar Asselin) aussi bien que du Parti conservateur (c'est le cas d'Arthur 
Beauchesne). Les seuls exclus, encore une fois, sont l'abbé Groulx, Henri Bourassa, et leurs partisans. Cet ostracisme, à en juger par la liste des collaborateurs du premier numéro, n'a cependant pas un caractère systématique : on y retrouve en effet le nom d'Ernest Bilodeau, alors correspondant du Devoir à Ottawa, et par ailleurs traducteur du Clash! de Moore. (Cela dit, sauf erreur, il ne semble pas que Bilodeau ait effectivement collaboré à la revue : c'est un des collaborateurs " virtuels ») À ceux qui s'étonnent ou s'alarment de l'éclectisme de la liste du premier numéro, la directrice répond que cette ouverture se veut conforme à l'usage "des grandes revues françaises ${ }^{28} »$. Le mois suivant, alors que la revue se trouve embarquée dans sa première polémique un peu violente, elle sent le besoin d'une mise au point supplémentaire : la revue veut être une tribune libre (c'est Madeleine qui souligne), ouverte à toutes les idées, et n'exerçant pas de censure sur les opinions de ses collaborateurs - une fois réservés, cela va de soi, les intérêts de la religion et de la morale ${ }^{29}$. Et comme les querelles journalistiques ont leurs dangers, elle en tire cette conclusion pratique : "Chaque collaborateur qui signe son article en assume l'entière responsabilité ${ }^{30}$."

En réalité, quelle qu'ait pu être l'intention initiale de Madeleine, il n'empêche que la revue ne se borna jamais à ce programme : dès le départ, elle fut autre chose que le simple espace neutre de discussions intellectuelles annoncé par la directrice. D'ailleurs, la dénonciation vigoureuse du nationalisme fauteur de désunion entre les « races » et l'ostracisme visant les chefs de file du nationalisme étaient des indices suffisamment clairs, qui ne pouvaient passer inaperçus du public. Les éditoriaux de Madeleine, les articles des collaborateurs les plus réguliers, mais surtout les premières querelles lancées par ces derniers allaient vite préciser le profil idéologique de la revue, dans laquelle on pourrait bientôt voir l'or- 
gane d'une faction bien définie : la bourgeoisie intellectuelle francophile indépendante du clergé, qui avait pris fortement position en faveur de l'engagement dans la guerre, qui s'était brouillé pour cela avec les nationalistes et qui depuis militait avec ardeur en faveur de la «bonne entente » et de «l'unité nationale ».

\section{Une première querelle}

L'analyse lucide faite quelques mois plus tard par un adversaire, au plus vif de la première grande querelle dans laquelle fut impliquée La Revue moderne, montre bien comment fut perçue l'entreprise de Madeleine :

Durant la guerre, Madeleine (c'est vous ${ }^{31}$ qui l'avez nommée) a versé dans une exagération que les vraies Canadiennes sauront souligner, elle a fait de la politique en attaquant les nationalistes de son mieux dans ses chroniques. Récemment, dans sa revue, elle a permis à des collaborateurs de nous fouailler, nous invitant à leur répondre chez elle. Les nationalistes ont leurs journaux. Quand ils sont attaqués, ils se défendent chez eux, ne fournissent pas de la copie à leurs ennemis. Ils ont refusé leur collaboration. Madeleine nous a alors piqués sournoisement dans de petites notes perfides. Drôle de revue neutre ${ }^{32}$ !

Malgré l'hostilité évidente de ce témoignage, la présentation des faits n'est guère contestable. Dès sa deuxième livraison (15 décembre 1919), La Revue moderne avait engagé les hostilités contre les nationalistes. Tout le numéro d'ailleurs pouvait donner à juste titre une impression de cohérence idéologique, dans le droit fil de l'idéal d'unité canadienne affiché dans l'éditorial-programme du mois précédent. Certes, la grande majorité des contributions (pages féminines ou culturelles, roman, etc.) n'avaient rien à voir avec la politique. Mais 
trois articles de fond, par des personnalités importantes, touchaient directement au thème de l'unité nationale et, donnant en quelque sorte le ton, venaient révéler l'orientation idéologique de la revue : il aurait été très difficile de croire qu'il n'y avait pas là un effort concerté. Disons à tout le moins que les nationalistes, pour leur part, n'en ont pas douté.

De ces trois articles de fond, le plus discret est un compte-rendu, par Louvigny de Montigny, du roman alors récemment paru de William Henry Moore, Polly Masson. Ce livre, aujourd'hui oublié, mériterait à lui seul une étude entière. Disons seulement qu'il s'agit d'un roman politique abordant, entre autres sujets, la mésentente entre les "races» au Canada. Tout comme L'Appel de la race, dont il préfigure en quelque sorte l'intrigue (quoique sous une forme inversée ${ }^{33}$ ), le roman de Moore transpose son sujet politique sur le plan privé, et le rapport entre les « races » est analysé à travers les relations d'un couple "mixte " : en l'occurrence un politicien francophobe voué à la cause de l'impérialisme britannique (William Larned) et, d'autre part, une jeune Canadienne française (la Polly ou Pauline Masson du titre), bien intégrée à la haute société anglaise, et dont Larned finit par tomber amoureux. Comme William Moore était l'un des plus notoires représentants de la "bonne entente » du côté canadien-anglais, il n'est pas étonnant qu'à la fin de son roman, le héros renonce à ses préjugés en même temps qu'à sa carrière politique : le livre s'achève en effet sur la demande en mariage que les lecteurs perspicaces pouvaient prévoir... Ce roman, même s'il n'eut pas le retentissement de Clash! et s'il ne fut pas traduit en français, fit néanmoins l'objet d'une large diffusion, recueillant des éloges aussi bien chez les nationalistes que chez les bonne ententistes ${ }^{34}$. Le livre de Moore n'était donc pas de nature à soulever la controverse, et le compterendu de Montigny n'aurait certes pas à lui seul déclenché les 
polémiques qui allait suivre ${ }^{35}$. Mais le lecteur nationaliste, peut être déjà agacé par l'éloge discret que Montigny adressait en passant à l'éducation pratique des collèges et universités d'Ontario, n'aurait pu être qu'encore plus irrité de la conclusion que ce dernier tirait de sa lecture de Moore :

[...] souhaitons qu'un écrivain de chez nous publie bientôt une réplique au Clash et démontre [...] aux barbiches soupçonneuses et aux moustaches [...] que, pour être de l'Ontario, nos compatriotes de langue anglaise et de religion protestante n'en possèdent pas moins des mérites et des qualités que nous aurions tout profit à acquérir ; et que cet écrivain leur fasse comprendre du coup que le préjugé est un vilain chiendent qu'il ne faut pas cultiver au nord de l'Outaouais, pendant qu'on s'efforce de l'extirper au sud.

Ce jour-là, moustaches émancipées et barbiches méfiantes renâcleront comme des ouistitis qu'on épuce ; mais la Bonne Entente aura fait encore - de Québec cette fois, un joli bout de chemin ${ }^{36}$.

Cette petite flèche lancée par Montigny à la fin de son compte rendu prenait une résonance spéciale du fait que celui-ci avait été précédé de deux autres articles importants explicitement consacrés à la «bonne entente». Le premier, de l'écrivain montréalais John Boyd, était donné directement en anglais, sans traduction ni explications ${ }^{37}$. Ce fait n'est pas unique dans l'histoire de la revue, mais demeure néanmoins singulier. On peut y voir d'abord le reflet d'une réalité sociale, à savoir que la bourgeoisie mondaine qui formait le lectorat de la revue pouvait fort vraisemblablement lire sans trop de difficultés un texte en anglais ${ }^{38}$. Mais dans le cas de l'article de Boyd, l'utilisation de la langue anglaise n'était pas non plus dépourvue d'implications symboliques, dans la mesure où il s'agissait d'en appeler à l'unité nationale. Boyd ici répond de toute évidence à une commande de Madeleine, et il peine un 
peu à remplir ses deux pages, malgré les longues citations de notables de toute sorte. Mais pour être juste, il faut reconnầtre que ces citations constituent précisément le fond de l'article : il s'agit de convaincre le lecteur de l'existence d'une bonne volonté au Canada anglais, de lui montrer qu'il y a des voix autorisées (au premier chef celle du Prince de Galles en personne) qui prônent la tolérance à l'égard du Canada français. Par ailleurs, la présence même de l'article dans la revue importe plus que tout ce que l'auteur pouvait dire. Notons seulement au passage que ce dernier n'entretenait pas le moindre doute sur l'orientation que Madeleine imprimerait à la revue : "La Revue moderne, it is most gratifying to know is to lend its efforts to the success of this great cause and for that alone, if for no other reason, it is entitled to the support of all patriotic Canadians $^{39}$. „ Il va de soi qu'un tel témoignage de bonne volonté, considéré isolément, n'avait rien pour choquer le plus sourcilleux des lecteurs ; tout au plus pouvait-il être reçu avec scepticisme par les nationalistes. Le texte de Boyd est donc surtout remarquable en ce qu'il contribue à définir l'esprit de la nouvelle revue. S'il pouvait prendre un sens moins innocent, c'est dans la mesure où il en accompagnait un autre qui cherchait plutôt à mettre le feu aux poudres. L'article qui eut un véritable éclat, en effet, c'est celui d'Arthur Beauchesne.

Beauchesne, tout comme Madeleine, est un autre de ces intellectuels méconnus du début du siècle ${ }^{40}$. Son éclectisme fait de lui une figure extrêmement curieuse, mais l'éparpillement de son activité explique aussi en grande partie l'oubli qui l'entoure aujourd'hui. Membre fondateur, au début de sa carrière, de la Ligue d'enseignement, membre également de la fameuse loge maçonnique "L'Émancipation" ${ }^{41}$ ", il s'attira l'hostilité de Bourassa et des milieux ecclésiastiques; il se signala d'ailleurs, comme journaliste, par des démêlés épiques avec $\mathrm{M}^{\mathrm{gr}}$ Bruchési. Publiciste pour le parti conservateur, can- 
didat malheureux de ce parti à plusieurs reprises, il fut récompensé en devenant, et pour très longtemps, assistant-greffier (puis greffier) de la Chambre des Communes - poste qu'il occupait au début des années vingt, alors qu'il collaborait régulièrement à La Revue moderne. Resté actif jusqu'à un âge avancé, il se mit au service de Duplessis dans les années cinquante, en qualité de conseiller spécial. Pour la postérité, Beauchesne demeure surtout l'auteur du manuel de procédure parlementaire qui porte son nom. Mais il a également écrit un ouvrage de critique littéraire qui n'est pas dépourvu d'intérêt, et qui témoigne d'une certaine liberté dans le jugement $^{42}$. Il constitue sans contredit l'une des figures-clefs du petit groupe réuni par Madeleine dans La Revue moderne.

C'est donc Beauchesne qui déclenche les hostilités. Son article, intitulé "Dissensions et rapprochement ${ }^{43}$ ", et qui devait en quelque sorte jouer le rôle de pendant français du texte de Boyd, se présente lui aussi comme un appel à la réconciliation des "races " et à l'unité nationale. Mais au contraire de Boyd, Beauchesne n'hésite pas à s'affranchir du langage diplomatique. Il commence son article par une analyse extrêmement lucide des divisions qui existent dans la Confédération. Allant au-delà de l'évidente opposition entre les éléments français et anglais, Beauchesne observe par exemple la différence importante de vues qui existe entre l'Ontario orangiste et francophobe (ce sont ses termes), et l'Ouest du pays, beaucoup plus marqué par la civilisation américaine et relativement indifférent à l'égard de l'Empire britannique. Mais tout en constatant la puissance des forces centrifuges ainsi que les inconvénients de la Constitution de 1867. pour la minorité canadienne-française, Beauchesne affirme (sans ressentir le besoin d'apporter de justifications) que la sécession est une impossibilité absolue. Du coup, il croit nécessaire la réconciliation des «races", et offre un programme à cet effet : appel 
générique à la bonne volonté anglo-saxonne, apprentissage approfondi de l'anglais pour la jeunesse canadienne-française. Sa proposition la plus originale est celle d'un manuel commun d'histoire du Canada, disponible dans les deux langues, mais offrant dans chaque cas la même perspective d'unité nationale sur tous les événements. Beauchesne admet que cela exigerait quelques omissions qu'il excuse d'avance pudiquement : "On sait que les traités scolaires ne sont jamais complets, qu'il sont rédigés dans le but de former la jeunesse. Plus tard, quand l'écolier aura fini ses humanités, il pourra approfondir les questions ${ }^{44}$. En somme, il s'agirait d'une sorte de censure de la vérité historique, mais justifiée par l'intérêt national : proposition qui, dans le cas présent, étonne surtout par sa franchise cynique. Évidemment, un tel programme avait déjà de quoi irriter considérablement l'opinion nationaliste. On imagine la tête de l'abbé Groulx, invité à participer à la rédaction de ce manuel d'histoire! Mais Beauchesne ne s'arrête pas là : il termine son article en attaquant nommément Bourassa. La stratégie polémique consiste ici à le rendre personnellement responsable de la francophobie orangiste et de l'aggravation des tensions :

La conduite de M. Bourassa, [...] ses articles et ses publications, depuis vingt ans, ont certainement accentué les préjugés des Anglo-Canadiens contre nous. [...] Une foule de choses quil a dites sont vraies, mais il a commis l'erreur de les dire très souvent en temps inopportun. [...] Quand nous préconisons devant les Ontariens la largeur d'esprit de nos compatriotes, on croit nous contredire victorieusement en nous lançant à la figure le nom de cet homme. Il faut que le genre Bourassa prenne fin si nous voulons atteindre l'union des races en ce pays ${ }^{45}$.

Après une telle déclaration de guerre, on admettra, comme le disait Pierre Dalbec, que La Revue moderne n'était plus guère 
en position d'être crue lorsqu'elle affichait ses prétentions $d$. neutralité.

Les réactions ne se firent guère attendre. Le dimanche qui suivit la parution du deuxième numéro de la revue, Le Nationaliste tomba sur Beauchesne à bras raccourcis, dans un article de deux colonnes en première page. On conçoit que le programme de «bonne entente » brossé par Beauchesne allait y faire l'objet de sarcasmes appuyés. De fait, il n'était pas difficile d'en souligner les naïvetés ou l'irréalisme:

Vous avez raison. Que tout aille bien et ça marchera. Que tous les morts ressuscitent, et il n'y aura plus de mois de novembre. Qu'on chauffe les rues, on pourra se promener en camisole. En un mot et pour aller plus vite : "Que la lumière soit!»

C'est le septième jour, Monsieur Beauchesne, vous pouvez vous reposer ${ }^{46}$ !

L'article, comme on le voit, brillait beaucoup plus par sa verve que par sa pensée, et l'auteur, Jean Mérolles, ne reculait pas devant des arguments (mais est-ce bien le mot juste ?) d'une nature plus personnelle:

Que n'écrivez-vous des contes champêtres, Beauchesne ? Vous avez un joli nom plein de frondaison, et, en littérature, la frondaison, n'est-ce pas le commencement de la sagesse?

$\mathrm{Si}$, comme écrivain canadien-français, vous avez du talent, il se peut faire aussi que, comme fonctionnaire du gouvernement, vous ayez des loisirs ${ }^{47}$.

Il ne semble pas que Beauchesne se soit abaissé à répondre à ces facéties de la jeunesse nationaliste - son statut de notable l'obligeant évidemment à un certain décorum. Mais cela ne l'empêcha pas pour autant de récidiver deux mois plus tard, et de donner encore plus d'ampleur à son attaque contre Bou- 
rassa - toujours selon la même stratégie rhétorique qui consistait à le rendre responsable de tous les reculs de la cause française :

À quoi nous a conduits le nationalisme après quinze ou vingt ans d'existence?

Avons-nous plus de prestige qu'autrefois dans l'administration fédérale?

Autrefois, il y avait des écoles où l'on enseignait le français d'une manière convenable dans le Manitoba et l'Ontario. Aujourd'hui, il n'y en a plus.

Autrefois, il y avait des ministres canadiens français [sic] dans le gouvernement de ces provinces. Aujourd'hui, il n'y en a plus.

Autrefois, il y avait trois ou quatre Canadiens français dans le cabinet fédéral. Aujourd'hui, il n'y en a qu'un seul, et il n'est pas député. [...]

Voilà comment notre influence périclite depuis que les nationalistes se sont constitués les prôneurs de notre peuple $^{48}$.

Prenant acte de ces attaques répétées, Le Nationaliste décide de mettre en cause non plus seulement Beauchesne, mais La Revue moderne elle-même dans la personne de sa directrice. L'article de Pierre Dalbec, "La Femme aux rubans ${ }^{49}$ ", rédigé sur un ton satirique, est une charge ad hominem contre Madeleine, ridiculisée pour son style, pour ses ambitions de «bas bleu », pour le "ruban » qu'elle dut recevoir vers cette époque de la France, pour sa revue, etc. C'est cet article qui donna lieu à l'intervention du $\mathrm{D}^{\mathrm{r}}$ Léo Pariseau, laquelle fut suivie de la réponse de Dalbec déjà citée ${ }^{50}$. Le débat entre les deux hommes n'est pas inintéressant: outre la question, centrale dans les circonstances, du féminisme ou de la place de la femme dans la société, on y voit également les désaccords profonds 
qui pouvaient exister entre nationalistes mêmes (puisqne Pariseau se dit tel $^{51}$ ) à propos de l'attitude qu'il convenart d'adopter quant à la France en guerre. Mais cette discussion sort un peu du cadre de la présente étude, dans la mesure où elle ne met pas directement en scène les acteurs de La Revue moderne.

Madeleine, toutefois, ne pouvait faire autrement que de réagir à ces éclats. Elle revient donc sur la querelle dans le numéro du 15 avril $^{52}$. Face aux outrances verbales du Nationaliste, il lui est facile de se donner le beau rôle et de prôner dans sa revue des débats plus élevés. Mais l'incident semble également lui avoir fait prendre conscience de sa responsabilité de directrice, ainsi que de l'urgence qu'il y avait de dissiper l'impression de partialité que les premières livraisons de la revue avaient pu créer:

Uné défense se pose [Madeleine veut sans doute dire : «s'impose "; à moins qu'elle ne veuille dire : « nous est proposée » ?] de la conduite de M. Bourassa et de ses disciples, défense faite par des amis sincères de notre œuvre. Je lui ouvre, avec empressement, les colonnes de la Revue moderne ${ }^{53}$.

C'était en effet la seule solution possible, dès lors que la revue souhaitait convaincre de son respect de «toutes les opinions sincères » et qu'elle entendait mettre en œuvre l'idéal de la tribune libre, qui jusqu'ici demeurait un vœu pieux. Des deux « amis sincères » qui vinrent enfin offrir leur concours à Madeleine pour défendre Bourassa, il en est un au moins qui mérite de retenir l'attention : il s'agit de Wilfrid Gascon ${ }^{54}$.

Né en 1870, encore actif jusque vers 1930 au moins, Wilfrid Gascon avait commencé sa carrière dans le journalisme sous la direction de Godfroy Langlois, mais ses opinions libérales radicales lui firent chercher la sécurité d'un poste de traducteur aux Communes (poste qu'il occupait en- 
core en 1920). Ce relatif retrait de la vie publique et le fait qu'il n'a pas écrit de livre expliquent qu'il soit aujourd'hui encore plus oublié que tous les écrivains mentionnés jusqu'à présent. Ses opinions pourtant ne sont pas sans originalité : bien que son libéralisme le place aux antipodes de Tardivel, Gascon fut avec lui l'une des rares personnalités de l'époque à défendre des thèses ouvertement indépendantistes. Ami proche d'Olivar Asselin dès le tournant du siècle, peut-être avaitil fait la connaissance de Madeleine par l'intermédiaire de ce dernier? Quoi qu'il en soit, c'est à lui que Madeleine confie le rôle d'avocat du diable ${ }^{55}$.

Gascon d'ailleurs ne s'acquittera que partiellement de ce rôle : s'il réfute avec vigueur les arguments de Beauchesne, il ne se déclare par pour autant favorable à Bourassa :

[...] M. Beauchesne et M. Bourassa sont tous deux d'accord pour s'accommoder du statu quo. Alors, qu'est-ce qui les divise ? Une simple question de tempérament. L'un est résigné, l'autre rebelle. L'un prie, l'autre crie. Mais sur le fond, ils forment une paire d'amis, si on peut dire ${ }^{56}$.

En effet, au contraire de Bourassa (et à plus forte raison de Beauchesne), Gascon ne croit pas possible la réconciliation entre la majorité et la minorité. Selon lui, l'hostilité de la majorité à l'égard des Canadiens français n'est pas l'effet d'une mauvaise humeur passagère, mais bien une politique systématique poursuivie depuis 1760. Comme on le conçoit, Gascon n'a aucune difficulté à trouver dans l'actualité des exemples pour appuyer sa thèse. Si Gascon souligne au passage que Beauchesne se montre injuste en faisant remonter à Bourassa la francophobie du Canada anglais, il n'en consacre pas moins la plus grande partie de son article à montrer les contradictions de la position bourassiste, et termine par un plaidoyer en faveur de l'indépendance. Comme on le voit, Made- 
leine avait trouvé le moyen de dissiper l'impression de partialité sans faire le moindre cadeau à son ennemi!

Du coup, la polémique se trouve relancée. D'autres acteurs interviendront (surtout du côté des opposants du nationalisme) ; mais c'est entre Beauchesne et Gascon que le dialogue est le plus intéressant. Jusqu'ici, Beauchesne n'avait rencontré (dans Le Nationaliste) que de jeunes disciples de Bourassa qui se divertissaient à ses dépens. Madeleine, en la personne de Gascon, lui offre un adversaire à sa mesure, lequel faisait avancer le débat, en le déplaçant sur la question de l'indépendance - enjeu peut-être très théorique, mais que la motion Francœur avait à tout le moins introduit dans le discours public deux ans plus tôt. Beauchesne entreprend donc d'argumenter pour montrer l'impossibilité de la solution prônée par Gascon ${ }^{57}$. Soixante ans avant le premier référendum, les lecteurs de la revue pouvaient donc lire des analyses pratiques qui semblent aujourd'hui d'une étonnante familiarité : considérations stratégiques, commerciales, psychologiques, problème de la répartition de la dette fédérale (alors de trois milliards de dollars...), estimation des coûts, situation des francophones hors Québec, etc. De toute cette argumentation, Beauchesne tire évidemment la conclusion que l'indépendance est absolument impraticable. Mais ce qui est le plus révélateur, c'est assurément sa réponse à une petite parabole que Gascon avait imaginée pour montrer l'absurdité de la position bonne ententiste:

[...] la bonne entente [disait Gascon], c'est ceci. Un voisin entré chez moi s'empare de ma montre. Survient un brave homme qui, ennemi du bruit, veut arranger l'affaire. D'abord, il me reproche de crier trop fort, puis se tournant vers le voleur, il lui demande gentiment : « Rendez-lui toujours sa chaîne et faites l'accord. » 
- Mais non, je veux ma montre et ma chaîne, et pour être sûr que je ne serai plus volé, je romprai avec un voisin aussi peu respectueux du bien d'autrui ${ }^{58}$.

À quoi Beauchesne répond :

Le brave homme peut avoir d'excellentes raisons de se montrer indulgent. Il craint probablement qu'une rupture complète entre vous et votre voisin n'ait des suites désastreuses. Vous ne pensez qu'à votre montre ; lui songe au péril dont vous seriez victime si vous encouriez la haine de ce voleur qui est capable d'incendier nuitamment votre maison, ruiner votre commerce et, par les intrigues que lui inspirera l'idée de vengeance, vous réduire à la misère noire. Dans l'intérêt de votre famille, vous feriez bien de vous contenter de la chaîne pour le moment, avec l'intention de mieux serrer votre bijouterie à l'avenirir ${ }^{59}$.

On remarquera que la vision du Canada anglais qui inspire la "contre-parabole » de Beauchesne est à tout le moins aussi noire, sinon plus, que celle que pouvaient s'en faire les nationalistes les plus convaincus. Comme on le voit, Beauchesne n'est pas aveuglé par son idéal de «bonne entente » au point de s'illusionner complètement sur les difficultés réelles de la réconciliation qu'il prône. Sa position ne paraît pas tant motivée non plus par une "anglophilie » inconditionnelle que par un calcul pragmatique des risques que peut faire courir une position nationaliste intransigeante. Il faut éviter ici tout anachronisme: Beauchesne, contrairement aux tenants actuels du fédéralisme, ne se préoccupe pas de dénoncer le nationalisme au nom d'un idéal éthique ou d'une inquiétude face aux dérives possibles des politiques identitaires. Sa position est véritablement celle d'un tacticien de la politique qui s'irrite non pas des idéaux de Bourassa (il en est au fond très proche : Bourassa aussi, au fond, souhaite la « bonne entente »...), mais de la tonalité combative de son discours. En somme, 
Beauchesne, haut fonctionnaire fédéral, parle en familier des salons de la bourgeoisie anglophone d'Ottawa, qui sait quel ton il faut adopter si l'on veut espérer être entendu de l'élite canadienne-anglaise. S'il est permis de risquer une généralisation, on pourrait dire que Beauchesne exprimait ici l'une des constantes idéologiques les plus durables au Canada français : l'aversion profonde pour la subversion et pour la confrontation violente. De ce point de vue, Gascon avait raison de dire que la distance de Beauchesne à Bourassa n'était pas si grande qu'il paraissait.

\section{$* * *$}

Cette première querelle de La Revue moderne, d'un caractère essentiellement antibourassiste, allait très rapidement déboucher sur une campagne systématique contre l'abbé Groulx qui, comme on sait, culminera par la querelle entourant L'Appel de la race. D'une certaine manière, cela reflète le contexte idéologique de l'époque : la revue apparait précisément au moment où Groulx et les intellectuels de L'Action française commencent à prendre leurs distances avec Bourassa - voire au moment où Groulx lui-même paraît sur le point de supplanter Bourassa, jugé alors trop "bonne ententiste", comme chef de file de la mouvance nationaliste. Mais à partir de ce débat sur l'unité nationale que La Revue moderne déclenche dès sa fondation, on peut tout de même tirer quelques conclusions. La motion Francœur pouvait bien, comme le suppose Yvan Lamonde, n'avoir été qu'un bluff de Lomer Gouin, destiné à frapper l'imagination du Canada anglais et à saper l'influence des nationalistes en ramenant l'opinion québécoise vers le Parti libéral du Québec ${ }^{60}$. Mais si la menace séparatiste de Francœur ne fut pas sérieuse, on voit qu'il n'en alla pas de même de tous les débats qu'elle put directement ou indirectement susciter. Deux ans après l'incident parlemen- 
taire, il est évident que la question est encore d'actualité. Suffisamment, en tout cas, pour que l'enjeu surgisse presque tout naturellement au cœur d'une polémique dont la doctrine de Bourassa (pourtant très éloigné lui-même du séparatisme!) avait été l'élément déclencheur ; suffisamment encore pour que l'ambitieuse revue de Madeleine devienne le lieu d'échanges qui préfigurent parfois étrangement ceux qui entourèrent les deux référendums. Si toutefois le débat nous parait remarquable, c'est en grande partie dans la mesure où il mettait en scène des interlocuteurs appartenant à ce qui a été appelé ici la bourgeoisie intellectuelle mondaine, indépendante du clergé (si ce n'est même anticléricale). La plupart des représentants de cette mouvance étaient allergiques au nationalisme, du moins tel qu'il s'incarnait dans la vie politique de l'époque, avec son visage bourassiste et anticonscriptionniste ${ }^{61}$; mais certains (tels Asselin ou Gascon) se définissaient d'une manière plus complexe par rapport à ce dernier. Les uns comme les autres étaient représentatifs d'une certaine modernité (comme l'annonçait justement le titre de la revue), et à ce titre, ils restaient sans doute bien minoritaires dans la société canadienne-française des années vingt ${ }^{62}$. Il n'est donc pas surprenant qu'ils aient été à peu près occultés par la figure imposante de Groulx qui, de fait, les supplanta complètement dans les années trente. Aucun de ces écrivains, aussi bien, n'était de taille à rivaliser avec l'abbé : entre l'intellectuel professionnel et les amateurs mondains, la partie n'était pas égale. Même la prolifique Madeleine fait figure de dilettante à côté de l'infatigable polygraphe qu'était Groulx. Mais peu importe cette disproportion entre les adversaires : notre compréhension du contexte intellectuel des années vingt serait faussée si nous négligions plus longtemps de tenir compte de ces voix qui, pendant un temps, ont donné la réplique au nationalisme et qui, de loin, ont annoncé les débats des années cinquante et soixante. 


\section{NOTES}

1 Pseudonyme de Mme Wilfrid Huguenin, née Anne-Marie Gleason, 18751943. À en juger d'après un autographe de 1938, celle-ci adoptait dans la vie privée la signature de « Madeleine Huguenin ». Elle sera cependant toujours désignée ici par le seul prénom de « Madeleine », conformément à son usage constant dans ses écrits publics.

${ }^{2}$ Chaque numéro reproduit le roman complet d'un auteur en vogue, le plus souvent français : par exemple Henry Bordeaux dans le premier numéro.

${ }^{3}$ François Ricard, «La Revue moderne: Deux Revues en une », Littératures, $\mathrm{n}^{\circ} 7$ (1991), pp. 77-84.

${ }^{4}$ Les résultats de leurs travaux ont été présentés au colloque « L'Artiste et ses lieux ", à Québec, en avril 2003, et ont été pris en compte dans la présente étude.

${ }^{5}$ Fernand Dumont et al., dirs., Idéologies au Canada français, tome II, 19001929, Québec, Presses de l'Université Laval, 1971, 377 p.

${ }^{6}$ Yvan Lamonde, Histoire sociale des idées au Québec (1896-1929), Montréal, Fides, 2004, pp. 94 et 223.

${ }^{7}$ Andrée Fortin, Passage de la modernité. Les Intellectuels québécois et leurs revues (1778-2004), $2^{c}$ édition, Québec, Presses de l'Université Laval, 2006. Cf. notamment ch. 7, « L'appel aux compétences », pp. 105-118.

${ }^{8}$ Madeleine, «S'unir pour grandir », La Revue moderne, 15 novembre 1919, pp. 8-9.

${ }^{9}$ Un exemple : paraphrasant Madeleine, Andrée Fortin dit que celle-ci envisage " la "haute destinée" du Canada français " (cf. Passage de la modernité, p. 113), alors que le texte de Madeleine (qu'Andrée Fortin cite pourtant juste avant) parle plutôt des « hautes destinées » de " notre pays » (il faut donc bien entendre : le Canada entier), invitant ensuite à l'union entre « les races, les classes, les groupes» («S'unir pour grandir », p. 8). Autre exemple : Andrée Fortin croit lire dans l'éditorial de Madeleine une affirmation de la « supériorité » de la culture canadienne-française (Passage de la modernité, pp. 113-114). Or, une lecture plus attentive du passage cité et de son contexte montre qu'il y a malentendu : Madeleine affirme plutôt l'égale dignité des héritages « latins » et « saxons ». Lorsqu'elle parle des « droits supérieurs » de la « famille française de ce continent ", on ne peut comprendre le passage que comme une affirmation des droits historiques découlant de l'ancienneté de la présence française en Amérique, qu'elle oppose à ceux qui inviteraient les Canadiens 
français à « renoncer à leur langue et à leur culte » («S'unir pour grandir », p. 9). C'est, si l'on veut, une forme de discours nationaliste minimal, mais tout à fait consensuel et « bonne ententiste » (le propos ne pouvant paraittre controversé que dans une perspective « orangiste »).

${ }^{10} \mathrm{Il}$ ne sera donc pas fait mention dans le présent article, sinon de manière allusive, à la campagne menée par La Revue moderne contre l'abbé Groulx, campagne culminant en 1922-1923 par la querelle de L'Appel de la race. Nous avons en effet consacré un article entier à cette affaire, auquel nous renvoyons le lecteur : cf. Jean-Christian Pleau, "Polémique sur un "mauvais livre”: L'Appel de la race de Lionel Groulx ", Voix et images, vol. XXVIII, $n^{\circ}$ 2-83 (hiver 2003), pp. 138-159. L'intention ici est plutôt de braquer le projecteur sur les premières polémiques de la revue avec les nationalistes, qui ont préparé le terrain pour l'affrontement subséquent avec Groulx.

${ }^{11}$ Vers 1924, Madeleine et sa revue connaissent divers problèmes auxquels il sera fait brièvement allusion plus loin. Le niveau intellectuel général de la revue en sera affecté, et celle-ci, avec le temps, se transformera de plus en plus en magazine populaire. L'espèce d'“ école » de La Revue moderne que nous entendons décrire dans le présent article n'aura existé que pendant très peu d'années.

${ }^{12}$ On reconnaîtra ici le titre d'un article d'Aurélien Boivin et de Kenneth Landry : "Françoise et Madeleine, pionnières du journalisme féminin au Québec ", Voix et Images, vol. IV, n 2 (décembre 1978), pp. 233-243. De Kenneth Landry, on trouve également quelques pages fort utiles sur Madeleine dans l'article "Premier Péché"), dans Maurice Lemire, dir., Dictionnaire des cuures littéraires du Québec, tome 2, 1900 à 1939, Montréal, Fides, 1980, pp. 909 912.

${ }^{13}$ Pour être exact, il conviendrait de mentionner des mémoires inédits : Mireille Éthier, Bio-bibliographie de Madeleine, École de bibliothéconomie, Université de Montréal, 1945 ; Juliette Plante, Madeleine, journaliste, Mémoire de maitrise, Université d'Ottawa, 1962, 121 p. ; Maria Eugenia de Matos-Andrade, Biographie et bibliographie descriptive de Madeleine, 1877-1943, Thèse de DES, Université de Montréal, 1970, 488 p. Ce dernier travail est entaché de naïvetés et d'insuffisances, et ne conserve d'utilité que par défaut. Le fait qu'on ne puisse citer de monographies plus professionnelles en dit long sur le statut marginal de Madeleine dans l'histoire littéraire québécoise.

${ }^{14}$ Madeleine a fait paraitre trois recueils d'articles : Premier Péché, Montréal, Imprimerie de La Patrie, 1902, 162 p. ; Le Long du chemin, Montréal, Imprimerie de la Patrie, 1912, 248 p. ; Le Meilleur de soi, Montréal, Éditions de La Revue 
moderne, 1924, 166 p.. Citons aussi une pièce de théâtre, En pleine gloire !Montréal, Compagnie de publication de La Patrie, 1919, 24 p., ainsi qu'un recuei de portraits biographiques (véritable bottin mondain) publié à la fin de sa vie : Portraits de femmes, Montréal, Éditions de La Patrie, 1938, 273 p. Mais, d'après le dénombrement d'E.M. de Matos-Andrade, Madeleine aurait écrit plus de trois mille articles.

${ }^{15} \mathrm{Cf}$. Valdombre (Claude-Henri Grignon), « Faits et gestes. Le Bas bleu sous l'orage ", Pamphlets de Valdombre, $4^{c}$ série, $\mathrm{n}^{\circ \mathrm{s}} 6$ et 7 , (novembre-décembre 1940), pp. 203-225. Cet article vitriolique est entaché d'une misogynie qui en rend la lecture aujourd'hui difficilement supportable. Il faut bien sûr tenir compte du fait que les outrances polémiques étaient inhérentes au style de Valdombre. Cela dit, Grignon lui-même devait sentir que son attaque contre Madeleine dépassait les bornes socialement acceptables en son temps, puisqu'il tâchait de se justifier en introduction. Notons tout de même que ses critiques ne relèvent pas toutes de cette obsession misogyne : sa colère tient d'abord aux positions politiques affichées par Madeleine, notamment à son rôle dans la querelle de L'Appel de la race; l'attaque misogyne est un moyen de disqualifier son adversaire.

${ }^{16}$ Des traditions orales rapportées dans le mémoire (hélas pas toujours fiable) de M. E. de Matos-Andrade, Biographie et bibliographie descriptive de Madeleine, laissent croire que Madeleine termina sa vie dans un isolement tragique. On soupçonne ici une certaine exagération. Des indices plus probants (notamment une dédicace autographe de Madeleine à un certain Bérubé, apparemment administrateur de ses biens, dans l'exemplaire de Portraits de femmes de la Bibliothèque nationale du Québec) permettent de formuler l'hypothèse suivante : ayant joui toute sa vie d'une aisance considérable (son mari, le $\mathrm{D}^{\mathrm{r}}$ Wilfrid Huguenin, avait hérité par sa mère de la fortune des seigneurs de Berthier), Madeleine fut considérablement gênée par la crise de 1929, et dut sans doute renoncer à son tôle d'animatrice mondaine (qui reposait évidemment d'abord sur sa position sociale privilégiée). On sait également, par les recherches du groupe de Laval (communication citée plus haut), que la situation financière de Madeleine s'était déjà considérablement détériorée dès la mort de son mari en 1924, et surtout après le vol spectaculaire de la caisse de La Revue moderne en 1926. (Il y eut un meurtre, et Madeleine, soupçonnée, fut contrainte de témoigner au procès.) Ces faits influencèrent sûrement sa décision de prendre sa retraite dès 1930 . À ces diverses épreuves il faut encore ajouter la mort prématurée de sa fille unique. C'est déjà un tableau suffisamment noir, mais rien ici n'expliquerait pourquoi Madeleine aurait soudainement été abandonnée de tous ses amis dans ses dernières années. Au con- 
traire : le sénateur Dandurand fait son éloge, on imprime une édition scolaire de ses Portraits de femmes, etc.

${ }^{17}$ La grande majorité des textes de Madeleine sont des écrits de circonstance, publiés dans la presse. Même sa pièce En pleine gloire ! fut conçue dans le cadre d'un événement mondain (la visite à Montréal d'un général français).

${ }^{18}$ Michel Lacroix, dans un article sur la mondanité littéraire à cette époque, avait déjà formulé l'hypothèse du rôle joué par certains salons montréalais dans la vie littéraire, et il mentionnait notamment le salon tenu par Madeleine. Cf. Michel Lacroix, « Des Montesquieu à Montréal : Le Nigog et la mondanité », Voix et images n 85, (automne 2003), pp. 105-114.

${ }^{19} \mathrm{Par}$ définition, la vie d'un salon mondain est une réalité difficile à cerner, même lorsqu'on peut s'appuyer sur des traces objectives - correspondances, mémoires, etc. Dans le cas du salon de Madeleine, celles-ci n'ont pas encore fait l'objet d'une enquête systématique. L'hypothèse présentée ici demanderait donc à être nuancée par une recherche plus approfondie. Notons ainsi que certaines des personnalités liées à La Revue moderne, en 1919, ne vivaient plus à Montréal, mais à Ottawa (ville où par ailleurs Madeleine avait vécu un temps). Ce qui paraît indéniable, c'est le rôle joué par Madeleine comme animatrice et pivot d'un réseau intellectuel.

${ }^{20}$ Sur Asselin en général, et sur ses rapports avec Madeleine et Montigny, on lira utilement la biographie monumentale d'Hélène Pelletier-Baillargeon, Olivar Asselin et son temps, Montréal, Fides, 1996 et 2004, 2 vol.

${ }^{21}$ Madeleine, «S'unir pour grandir», p. 8.

${ }^{22}$ Le terme de « race » sera ici utilisé conformément à l'usage canadien des années vingt, mais toujours entre guillemets. Sans revenir sur des débats bien connus, il n'est sans doute pas inutile de rappeler que ce terme, très courant à l'époque aussi bien en français qu'en anglais, s'employait dans un sens beaucoup plus large qu'aujourd'hui, recouvrant en gros les notions actuelles d'« ethnie » ou de " nationalité ». En fait, le mot renvoyait autant sinon plus à l'identité culturelle qu'à l'hérédité physique - encore qu'il paraisse difficile de nier dans le concept la présence d'une vague dimension biologique. Notons simplement que le terme ne provoquait ni malaise ni malentendu, et que dans bien des cas, il était employé par des gens qui ne s'intéressaient ni de près ni de loin aux théories racistes sur l'hérédité qui avaient cours à l'époque.

${ }^{23}$ Sur ces éléments du contexte politique, et notamment sur la motion Francœur, on relira utilement la synthèse présentée dans le premier chapitre de l'Histoire sociale des idées au Québec (1896-1929), d'Yvan Lamonde, pp. 43-44. 
${ }^{24}$ Madeleine, «S'unir pour grandir», p. 8.

${ }^{25}$ «Une mise au point », La Revue moderne, 15 février 1920, p. 39. Cette note de la rédaction n'est pas signée, mais il est raisonnable de supposer que Madeleine en assume la responsabilité. Par ailleurs, Madeleine elle-même mentionne l'appui financier apporté par son mari lors du lancement de la revue (cf. "Notre anniversaire », La Revue moderne, novembre 1925, p. 11). Notons que s'il y a indépendance, c'est par rapport au monde de la politique partisane : la publicité commerciale, elle, joue un rôle essentiel dans la revue (jusqu'à influencer le contenu éditorial, notamment dans les pages féminines). Rappelons qu'après la mort du $\mathrm{D}^{\mathrm{r}}$ Huguenin en 1924, la situation financière de la revue devint plus précaire (cf. note 16).

${ }^{26}$ Madeleine, «S'unir pour grandir », p. 8. C'est nous qui soulignons.

${ }^{27}$ William Henry Moore (1872-1960), politicien et écrivain canadien-anglais. Auteur d'un ouvrage très remarqué sur la querelle scolaire franco-ontarienne et sur les rapports entre Canadiens anglais et Canadiens français (The Clash! $A$ Study in Nationalities, Londres, Dent, 1918), il est unanimement célébré au Canada français (et notamment au Devoir) comme sincère défenseur des droits des francophones. Il est également l'auteur d'un roman politique sur le même sujet, Polly Masson (Londres, Dent, 1919). John Boyd (1864-1933), personnalité publique montréalaise, auteur d'un ouvrage poétique en langue anglaise sur la mort de Dollard, mais surtout de brochures (toujours en anglais) sur le nationalisme canadien-français, sur les rapports entre francophones et anglophones ; il est connu, lui aussi, comme un partisan de la bonne entente. Boyd publia un article sur l'unité canadienne dans la seconde livraison de La Revue moderne ("The Secret of National Unity », 15 déc. 1919, pp. 15-16).

${ }^{28}$ La Revue moderne, 15 janvier 1920, p. 26.

${ }^{29}$ Cette exception affichée en faveur de l'orthodoxie religieuse est une sorte de bienséance obligatoire, dans le contexte de 1920. Cela dit, La Revue moderne était bel et bien indépendante du clergé, et plusieurs de ses collaborateurs se tenaient à une certaine distance de l'Église, au point de pouvoir passer pour suspects aux yeux des censeurs les plus intransigeants : c'était notamment le cas de Montigny et de Beauchesne.

${ }^{30}$ «Une mise au point », p. 39.

${ }^{31}$ L'auteur, Pierre Dalbec, s'adresse ici personnellement au D ${ }^{r}$ Léo Pariseau, qui avait vigoureusement défendu Madeleine contre ses adversaires nationalistes. Notons que Pariseau n'est pas un des proches de Madeleine (il dit ne pas la connaitre), et qu'il ne figure pas non plus dans la fameuse liste des 
collaborateurs de La Revue moderne. Son intervention dans le débat a toutes les apparences d'une initiative spontanée.

${ }^{32}$ Pierre Dalbec, «Réponse de Pierre Dalbec au $\mathrm{D}^{\mathrm{r}}$ Léo Parizeau [sic] », Le Nationaliste, 14 mars 1920, p. 1.

${ }^{33} \mathrm{La}$ question se pose de savoir si Groulx avait lu le roman de Moore. Toutefois, il ne pouvait guère faire autrement que d'en avoir entendu parler. Le projet de L'Appel de la race pourrait donc lui être venu comme celui d'une réponse à une œuvre dont il désapprouvait le message et l'influence. L'hypothèse est séduisante, mais demanderait évidemment à être étayée plus solidement.

${ }^{34}$ Le livre fut notamment diffusé par la librairie du Devoir, qui l'annonçait régulièrement en bas de page et lui consacra un éditorial élogieux (cf. Georges Pelletier, "Un roman politique canadien », Le Devoir, 23 octobre 1919, p. 1).

${ }^{35}$ Sauf erreur, aucun nationaliste ne crut bon de répondre à Montigny, et ce dernier s'abstint d'intervenir dans les polémiques sur l'unité nationale qui allaient suivre. C'est un peu plus tard, lors de la querelle de L'Appel de la race, que Montigny jouerait un rôle de premier plan.

${ }^{36}$ Louvigny de Montigny, "Polly Masson», La Revue moderne, 15 décembre 1919, pp. 21-23.

${ }^{37}$ Boyd, «The Secret of National Unity », pp. 15-16.

${ }^{38}$ Pour un autre exemple d'article exceptionnellement publié en anglais par la revue, cf. Donald Downie, "Prussian Bluff. The Last Phase ", La Revue moderne, mars 1923, pp. 17-18. Encore une fois, aucune justification n'est donnée de cette entorse au caractère francophone de la revue. Il faut supposer qu'elle s'explique seulement par le côté prestigieux de la contribution (Downie est présenté comme " formerly publisher of the Paris International Courier»). Notons au passage qu'à la même époque, dans un journal comme Le Nationaliste, il y avait aussi une brève section en anglais, consacrée aux événements d'Irlande. Cela pouvait s'expliquer alors comme une forme de courtoisie entre nationalistes unis dans un même combat contre l'impérialisme britannique ; mais il est à tout le moins intéressant de noter ces rapports amicaux entre nationalistes canadiens-français et nationalistes irlando-montréalais d'expression anglaise. On sait que dans d'autres circonstances (notamment la querelle scolaire ontarienne), Canadiens français et Irlandais catholiques s'étaient trouvés divisés.

${ }^{39}$ Boyd, "The Secret of National Unity », p. 16. 
${ }^{40}$ Né en 1876, mort en 1959. Une seule étude d'un peu d'envergure lui a ét consacrée : Gary Levy, « La Vie de Beauchesne », Revue parlementaire canadienii, printemps 1985 , pp. 12-16 ; été 1985, pp. 11-15 ; automne 1985, pp. 16-17; hiver 1985-1986, pp. 2-5.

${ }^{41}$ Cette loge, on le sait, rassemblait quelques-unes des personnalités les plus en vue du libéralisme anticlérical. Voir à ce sujet Roger Le Moine, « Le Grand Orient de France dans le contexte québécois (1896-1923)", dans Yvan Lamonde, dir., Combats libéraux au tournant $d u X X^{*}$ siècle, Saint-Laurent, Fides, 1995, pp. 145-157.

${ }^{42}$ Arthur Beauchesne, Écrivains d'autrefois, Ottawa, Mortimer, 1930, 316 p. L'ouvrage est composé d'une série d'essais, sur Napoléon, Stendhal, Flaubert, les Goncourt, Barbey d'Aurevilly, Talleyrand. Bien que Beauchesne fasse preuve d'un goût qui paraittrait aujourd'hui incroyablement timoré, ses choix de lectures n'en sont pas moins remarquables. Il est intéressant aussi de noter qu'il lui arrive de citer tranquillement Renan ou Anatole France sans autrement songer à les blâmer (tous deux étaient, on le sait, des penseurs anticléricaux des plus notoires). Bourassa pouvait avoir raison de se méfier de lui...

${ }^{43}$ Arthur Beauchesne, « Dissensions et rapprochement », La Revue moderne, 15 décembre 1919, pp. 17-18.

${ }^{44}$ Ibid., p. 18.

${ }^{45}$ Ibid., p. 18.

${ }^{46}$ Jean Mérolles, « M. Beauchesne a tout réglé », Le Nationaliste, 21 décembre 1919, p. 1.

\section{${ }^{47}$ Ibid.}

${ }^{48}$ Arthur Beauchesne, "C'est assez d'anglophobie ", La Revue moderne, 15 février 1920, p. 18.

${ }^{49}$ Pierre Dalbec, « La femme aux rubans », Le Nationaliste, 29 février 1920, p. 1.

${ }^{50}$ Léo-E. Pariseau, "À propos de la "Femme aux rubans" ", Le Nationaliste, 7 mars 1920 , p. 2. ; Dalbec, «Réponse de Pierre Dalbec au D $D^{r}$ Léo Parizeau [sic] ».

${ }^{51}$ « Je suis ardemment nationaliste et résolument autonomiste. Mais, comme Asselin et bien d'autres, j'ai préféré faire de ma doctrine une voile pour accélérer ma traversée des mers. " (Pariseau, "À propos de la "Femme aux rubans" $)$. 
${ }^{52}$ Madeleine, " Nos méthodes et les autres... », La Revue moderne, 15 avril 1920, p. 11.

${ }^{53}$ Ibid.

${ }^{54}$ Wilfrid Gascon, « La bonne entente par le "self determination" ", La Revue moderne, 15 avril 1920, pp. 15-16. L'autre " ami sincère » ne signa qu'un article court, sous le nom vraisemblablement supposé de "Jean Deslys ».

${ }^{55}$ Ces divers renseignements sur Gascon sont tirés de la biographie d'Olivar Asselin par Hélène Pelletier-Baillargeon, passim, mais aussi des séries annuelles des Biographies canadiennes-françaises de Raphaël Ouimet pour la période des années vingt.

${ }^{56}$ Gascon, «La bonne entente par le "self determination” », p. 15.

${ }^{57}$ Arthur Beauchesne, « Pourquoi l'indépendance », La Revue moderne, 15 juin 1920, pp. 15-16.

${ }^{58}$ Gascon, «La bonne entente par le "self determination" », p. 16.

${ }^{59}$ Beauchesne, « Pourquoi l'indépendance », p. 15.

${ }^{60} \mathrm{Cf}$ Lamonde, Histoire sociale des idées an Québec (1896-1929), pp. 43-44.

${ }^{61}$ Il faut en effet rappeler que les Madeleine ou Montigny avaient contribué au Nationaliste vers le temps de sa fondation par Asselin! Le jeune Beauchesne aussi, vers cette époque, avait défendu des idées nationalistes ; et dans les années cinquante, il allait être attiré par les positions autonomistes de Duplessis.

${ }^{62}$ On peut d'ailleurs noter que beaucoup des écrivains cités dans le présent article se situaient en quelque sorte dans les marges de la société canadiennefrançaise, et gravitaient autour du monde anglophone : Montigny, Gascon, tous deux traducteurs au Parlement, étaient appelés à fréquenter des milieux anglo-saxons. C'était aussi la situation de Beauchesne, avec cette différence que son poste était encore plus prestigieux et lui ouvrait la plus haute société. $\mathrm{Du}$ Roure, pour sa part, était professeur à McGill, et l'on sait par divers témoignages qu'il était très apprécié dans la bourgeoisie anglo-montréalaise. Sur ce dernier point, cf. Pleau, « Polémique sur un "mauvais livre”... » 